\title{
Microscopio simple: mucho más que una simple lupa
}

\author{
Alejandro del Mazo Vivar \\ I.E.S. Francisco Salinas.Salamanca.España.marovivar@gmail.com \\ ORCID:https:/ / orcid.org/0000-0002-8611-2585
}

[Recibido: 29 Septiembre 2018. Revisado: 26 Noviembre 2018. Aceptado: 26 Diciembre 2018]

Resumen: El microscopio simple fue durante mucho tiempo el mejor instrumento con que se contó para escudriñar el mundo microscópico. Su calidad es superior a la que se le atribuye normalmente y permite observar casi todas las estructuras y orgánulos que se ven con un moderno microscopio compuesto. Se explicará la forma de construir un potente microscopio simple con más de 400 aumentos, usando una microesfera de vidrio.

Palabras clave: Microscopio simple; Lupa; Lente de bola o esférica; Distancia focal; Aumento; Resolución.

Simple microscope: much more than a simple magnifying glass

Abstract: The simple microscope was for a long time the best instrument to scrutinize the microscopic world. Its quality is greater than what is usually assumed and it allows the observation of almost all structures that are seen with a modern compound microscope. We will explain how to build a powerful simple microscope with more than 400 magnification, using a microsphere lens.

Keywords: Simple microscope; Magnifying glass; Ball lens; Focal length; Magnification; Resolution.

Para citar este artículo: Del Mazo, A. (2019) Microscopio simple: mucho más que una simple lupa. Revista Eureka sobre Enseñanza y Divulgación de las Ciencias 16(2), 2401 . doi: 10.25267/Rev_Eureka_ensen_divulg_cienc.2019.v16.i2.2401

\section{Introducción}

Se encuentra bastante extendida la idea de que el microscopio simple ha sido poco más que una lupa de disección con la que lograr aumentos modestos y con una calidad de imagen muy inferior a la de los microscopios compuestos. Sin embargo, la realidad es bastante diferente.

En la fabricación de las pinturas reflectantes utilizadas en señalización vial o para recubrimiento de pantallas de proyección se emplean microesferas de vidrio, con un diámetro inferior a $1 \mathrm{~mm}$, que suministran algunos comercios de material para obras públicas (figura 1). Esas microesferas pueden usarse también como lentes para fabricar microscopios simples con aumentos comparables a los de los objetivos de alta potencia de un moderno microscopio compuesto.

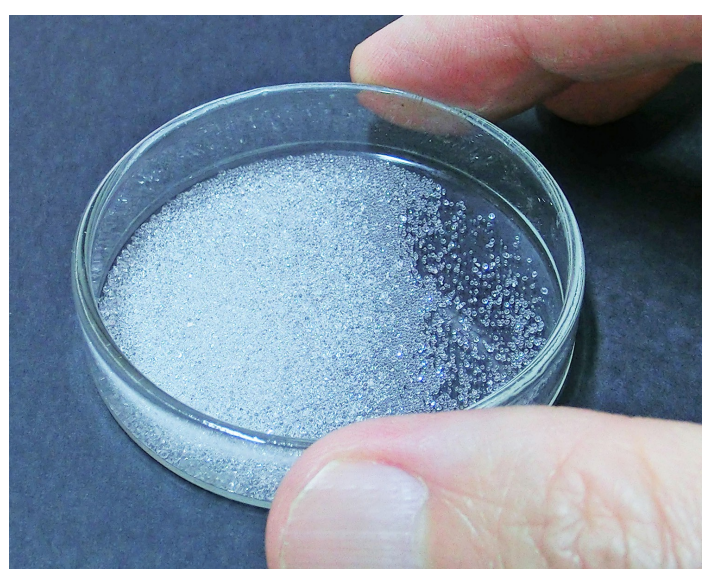

Figura 1. Microesferas de vidrio utilizadas para pinturas reflectantes.

\section{Los primeros instrumentos de aumento}

La posibilidad de obtener imágenes aumentadas usando esferas y lentes de vidrio había sido reconocida ya por Alhazen en el siglo XI y por Roger Bacon en el siglo XIII (Snyder 2017). 
Mucho más tarde, utilizando precisamente lentes de aumento, el artista Joris Hoefnagel realizó detalladas pinturas de insectos y plantas en la segunda mitad del siglo XVI (Stafford 2001).

El microscopio compuesto por dos lentes se comenzó a utilizar en las primeras décadas del siglo XVII, de forma paralela al telescopio. Dibujos de la abeja realizadas por Federico Cesi y Francesco Stelluti, publicadas en 1625, forman parte del primer estudio conocido que se realizó con la ayuda del microscopio compuesto. El creciente interés por las posibilidades de este instrumento llevaron a Robert Hooke (1989) a iniciar, bajo la sugerencia de la Royal Society de Londres, un ambicioso estudio microscópico que ofrecía por primera vez una visión desconocida de la fauna y la flora. Sus observaciones, trasladadas a unos excelentes grabados, fueron recogidas en 1665 en su Micrographia, un libro que alcanzó extraordinario éxito. El microscopio compuesto de Hooke proporcionaba alrededor de 20 aumentos y, aunque en el prefacio de su obra hablaba de los grandes aumentos que podrían proporcionar microscopios simples que utilizaran perlas de vidrio, las descartó por las dificultades que presentaba su uso.

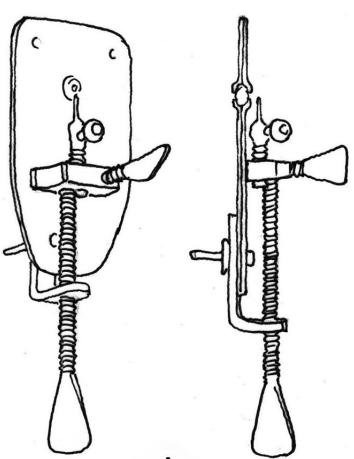

A

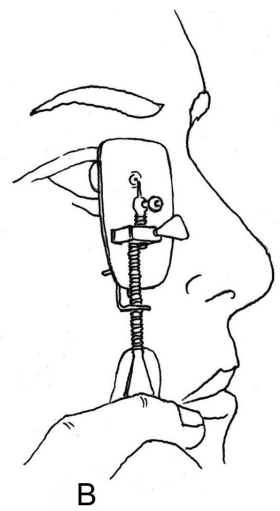

Figura 2. (A) Microscopio de Leeuwenhoek que se halla en la Universidad de Utrecht. Es el más potente de los pocos que se conservan actualmente. (B) Forma de usar un microscopio de Leeuwenhoek.

lecuwenhoek era un comerciante de paños establecido en Deft que, sin abandonar su profesión, en algún momento de su vida comenzó a construir pequeños microscopios simples. Parece que llegó a fabricar en torno a quinientos, aunque en la actualidad solo se conservan diez, uno sin lente (Robertson 2015). El más potente de ellos, que se encuentra en la universidad de Utrecht, tiene 266 aumentos (figura 2). Leeuwenhoek afirmó que fabricaba lentes desde 1659, sin que esté claro cómo aprendió a construirlas (Snyder 2017), ya que siempre guardó celosamente el secreto de las técnicas usadas. Los exámenes realizados a las lentes de sus microscopios, hasta donde es posible, hacen pensar que usó más de un procedimiento.

Conviene comparar las características del microscopio compuesto de Hooke con las de los microscopios simples de Leeuwenhoek. El de Hooke, con aproximadamente 20 aumentos, lo que equivale a la baja potencia de un microscopio moderno, estaba muy limitado porque la combinación de lentes sin corregir incrementa los efectos debidos a las aberraciones ópticas. Los microscopios de Leeuwenhoek utilizaban una sola lente y llegaron a multiplicar por más de diez los aumentos del utilizado por Hooke, con una buena calidad de imagen. En la práctica, la diferencia entre tales instrumentos significa que Hooke podía ver los detalles de un piojo, pero era imposible que pudiera 
observar bacterias, mientras que Leeuwenhoek sí las vio; de hecho fue la primera persona que logró observarlas.

Las investigaciones de Zuylen (1981) y de Ford (1985) ofrecen conclusiones bastante coincidentes acerca de las características de las lentes de los microscopios de Leeuwenhoek y constatan su alta calidad. Con estos resultados, Ford rebate las falsas ideas aceptadas acerca de los microscopios simples y expone algunas conclusiones:

- En los microscopios compuestos, a menos que el fabricante esté en condiciones de equilibrar los componentes ópticos, las aberraciones ópticas se incrementarán. Por esta razón, hasta la segunda mitad del siglo XIX, cuando las lentes acromáticas alcanzaron suficiente calidad, no fue posible diseñar microscopios compuestos que superaran a los simples.

- Se dice que los microscopios simples tenían limitado su uso por culpa de las aberraciones, cromática y esférica, por lo que daban imágenes coloreadas y borrosas. La realidad es que estos microscopios proporcionaban imágenes que podían compararse favorablemente con las de los modernos microscopios.

- Existe la creencia de que hasta la aparición de los microscopios acromáticos no se hicieron hallazgos de importancia en microbiología. Sin embargo, los descubrimientos de las bacterias, los espermatozoides, el núcleo celular, así como el movimiento browniano, se hicieron con microscopios simples.

- La sencillez técnica de los microscopios simples los hace ideales como instrumentos de campo y para la enseñanza.

El microscopio simple fue muy usado por los naturalistas durante las primeras décadas del siglo XIX. Robert Brown descubrió el núcleo celular y observó el movimiento que lleva su nombre -movimiento browniano- con un microscopio de este tipo (Brown 1828). Además, recomendó uno de estos instrumentos a Charles Darwin antes de que este iniciara su viaje en el Beagle. Estos aparatos eran de Robert Bancks, un importante fabricante que construía excelentes microscopios, dotados con juegos de lentes que abarcaban desde unos 20 aumentos hasta 160 aumentos (figura 3).

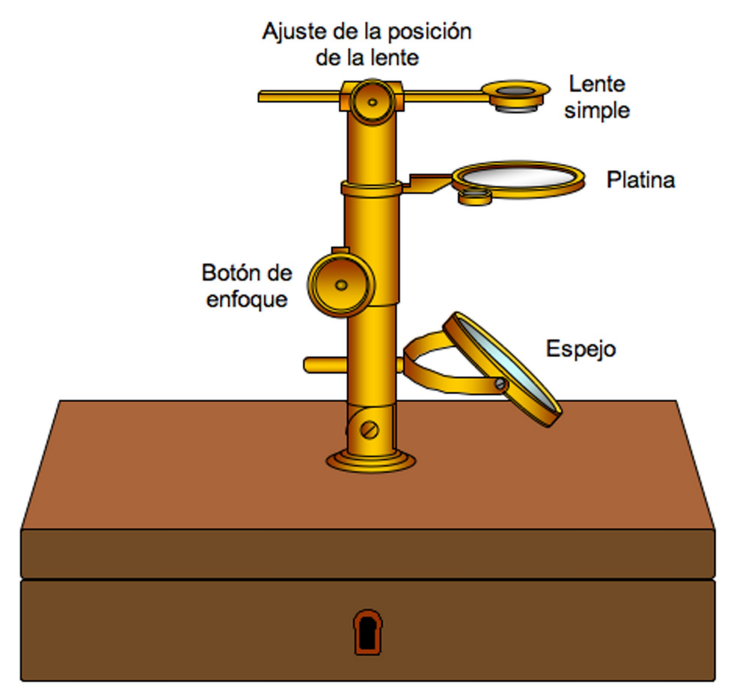

Figura 3. Uno de los microscopios simples de Robert Brown, construido en las primeras décadas del siglo XIX por Robert Banks.

\section{Fundamento de la lupa y del microscopio simple}

Tanto el microscopio simple como la lupa, que se puede considerar un microscopio simple de pocos aumentos, constan de una lente convergente. Cuando el objeto a observar se sitúa a una distancia de la lente que es inferior a su distancia focal, el instrumento produce una imagen virtual, derecha y de mayor tamaño que el objeto (figura 4, A).

$\mathrm{El}$ aumento que proporciona una lente convergente es inversamente proporcional a su distancia focal y esta se encuentra relacionada con el radio de curvatura de sus caras. Cuanto más pequeña sea una lente y más curvatura tengan sus caras mayor aumento producirá cuando se utilice como lupa. 

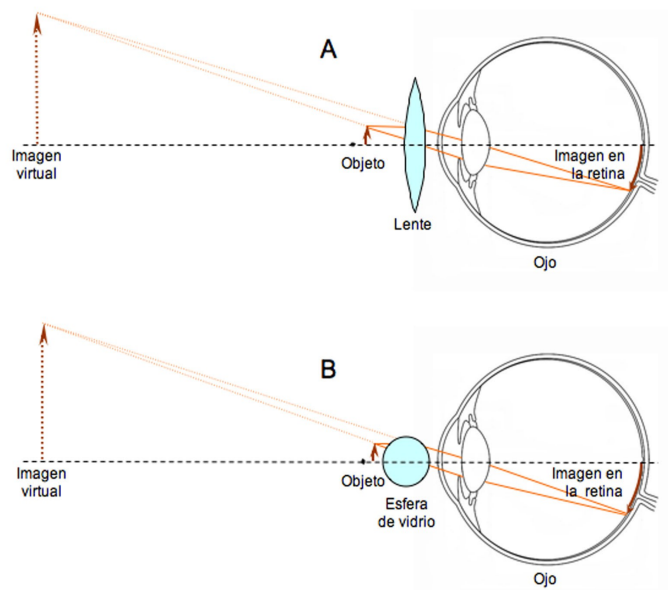

Figura 4. (A) Lente convergente que actúa como lupa. (B) Esfera de vidrio que actúa como lupa.
Una esfera transparente de vidrio cumple las condiciones de una lente convergente, por lo que puede utilizarse como una lupa. Si se cuenta con una esfera de diámetro muy pequeño, el aumento que se consiga con ella será importante (figura 4, B).

\section{Construcción de un microscopio simple}

Es necesario advertir que los microscopios de Leeuwenhoek que se conservan y los utilizados por Brown emplean lentes pulidas biconvexas, pero no esferas de vidrio. Sin embargo, el uso de una microesfera, como se hará aquí, permite la construcción de un microscopio cuyo aumento es superior al de cualquiera de los antiguos microscopios simples que se conservan en la actualidad.

Las microesferas de vidrio empleadas para pinturas reflectantes pueden utilizarse como lentes. Para construir un microscopio de muchos aumentos se selecciona una, de $0,8 \mathrm{~mm}$ de diámetro (medida con un microscopio compuesto que está dotado de ocular con retícula calibrada) y se limpia cuidadosamente con líquido para limpieza de óptica.

Para que una diminuta esfera de vidrio, con los elevados aumentos que proporciona, resulte útil, el microscopio tiene que disponer de un sistema mecánico suficientemente preciso. Aquí se ha elegido un diseño que funciona de forma muy satisfactoria, aunque pueden idearse otros. Se cortan dos láminas rectangulares de aluminio de $2,0 \mathrm{~cm}$ por $1,2 \mathrm{~cm}$ y $0,15 \mathrm{~mm}$ de espesor cada una. Ambas láminas se perforan con una broca de $0,6 \mathrm{~mm}$ de diámetro y se eliminan las rebabas con lija fina de agua del número 360, por ejemplo (figura $5, \mathrm{~A}$ ). En la zona perforada de cada lámina se crea una pequeña depresión, de modo que entre ambas quede aprisionada la lente (figura 5, B). Por último, las dos láminas se inmovilizan (figura $5, \mathrm{C}$ ) con un poco de adhesivo epoxi de dos componentes. Es muy importante que la lente sobresalga de la superficie del aluminio por el lado dirigido hacia la preparación microscópica que va a observarse (figura $5, \mathrm{D}$ ).
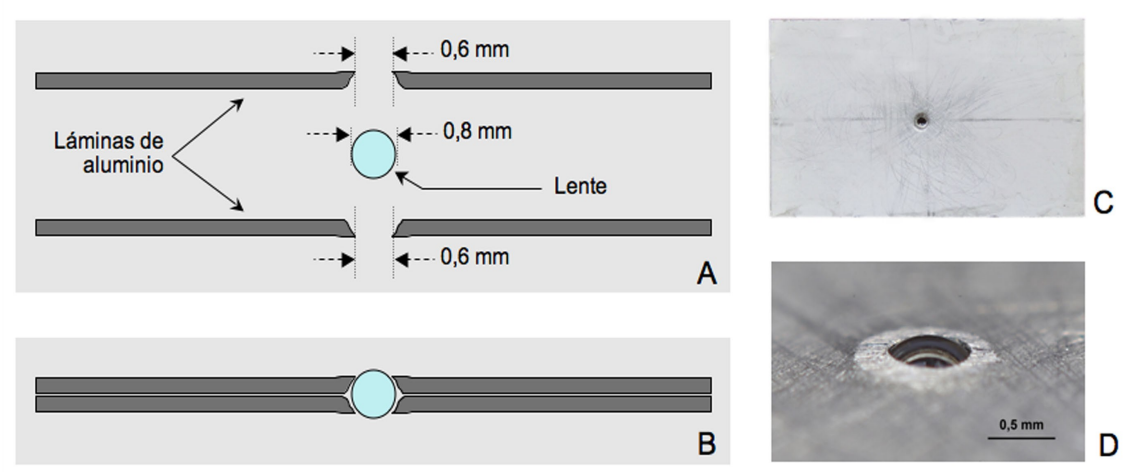

Figura 5. (A) Dos láminas de aluminio de iguales dimensiones se perforan para poder alojar entre ellas la lente. (B) Lente aprisionada entre las láminas de aluminio. (C) Fotografía en la que se observa la lámina de aluminio, del lado dirigido hacia la preparación microscópica. (D) Macrofotografía que muestra la lente, del lado que aparece en la imagen anterior. Se observa cómo la lente sobresale de la lámina de aluminio.

El bloque de láminas que alberga la lente ha de fijarse a un soporte suficientemente sólido. En este caso, se ha pegado sobre la abertura central realizada en una placa rígida de aluminio que puede girar sobre una bisagra (figura 6, A). El portaobjetos con la muestra se deposita sobre una platina, que puede ser de madera. La lente tiene que estar a una distancia de la muestra inferior a 0,2 $\mathrm{mm}$, por lo que es 
necesario un sistema de enfoque bastante preciso; un tornillo que gira en el interior de una tuerca pegada a la platina cumple satisfactoriamente este requisito. La mejor calidad de la imagen se consigue iluminando la muestra con una fuente extensa como es el cielo (evitando la luz directa del Sol) cuya luz se dirige por medio de un pequeño espejo giratorio, que puede ser plano o, mejor, cóncavo. La observación se realiza aproximando mucho el ojo a la lente (figura 6, B).

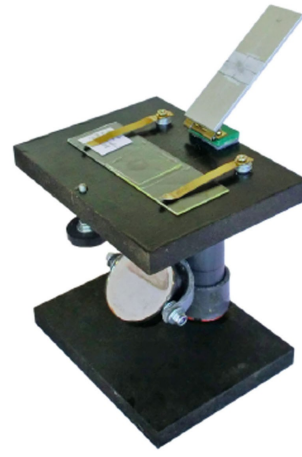

A

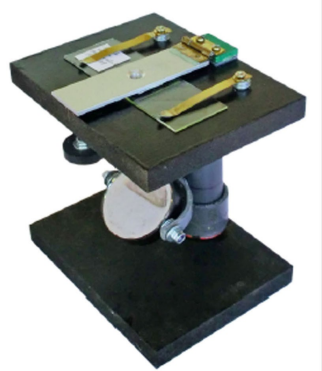

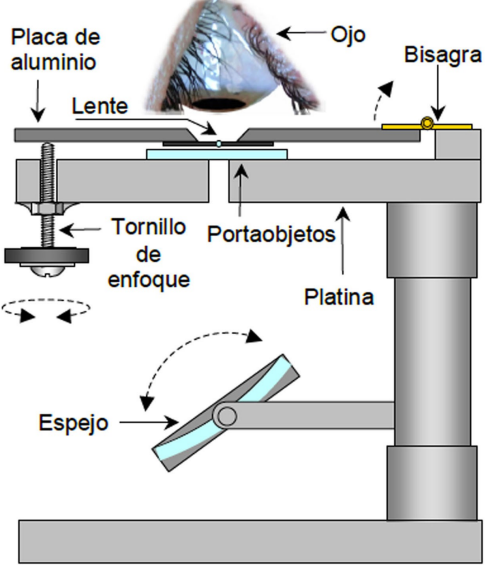

Figura 6. (A) Fotografía del microscopio simple con todos sus elementos. En particular, se observa el conjunto de láminas de aluminio que contiene la lente, pegado bajo la abertura central realizada en una placa rígida de aluminio que gira sobre una bisagra. (B) Dibujo que muestra las partes del microscopio simple.

\section{Parámetros de la lente}

El elemento fundamental del microscopio es una lente de bola (figura 7). Al aplicar la ecuación de una lente gruesa (Hecht 2000) a una esfera de diámetro $D$ e índice de refracción $n$, que se encuentra en aire, la distancia focal, $f$, es:

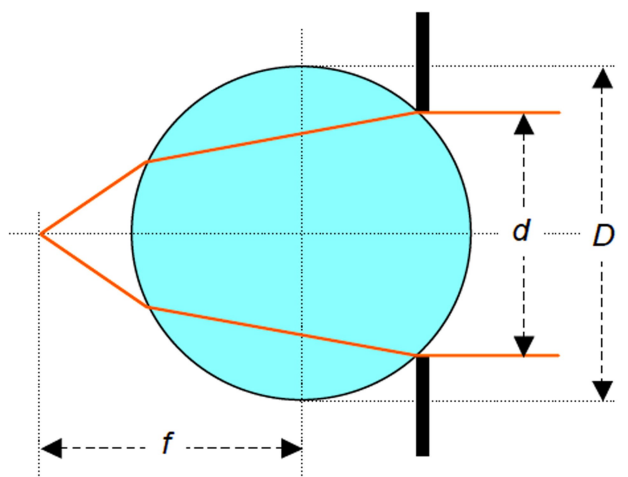

Figura 7. Algunos parámetros de una lente de bola: $f$, distancia focal; $D$, diámetro de la esfera; $d$, diámetro de la pupila de salida.

$$
f=\frac{n D}{4(n-1)} .
$$

Hay que tener presente que la distancia focal objeto y la distancia focal imagen tienen el mismo valor absoluto, que se miden desde los correspondientes puntos principales y que, en este caso, ambos se encuentran en el centro de la esfera.

El aumento angular, $M$, es el producto de la potencia de la lente, $1 / f$, por la distancia al punto próximo, $d_{0}$ :

$$
M=\frac{d_{0}}{f} .
$$

La resolución de la lente, según el criterio de Rayleigh, viene dada por el radio de Airy, $r_{\mathrm{A}}$ :

$$
r_{\mathrm{A}}=\frac{1,22 \lambda f}{d}
$$

donde $\lambda$ es la longitud de onda de la luz utilizada y $d$ el diámetro de la pupila de salida.

La esfera utilizada tiene un diámetro de 0,8 mm, un índice de refracción de 1,5 (es de vidrio sódicocálcico, utilizado para envases y ventanas, cuyo índice de refracción está comprendido entre 1,51 y 1,52), y está montada entre láminas de aluminio con aberturas circulares de $0,6 \mathrm{~mm}$ de diámetro. Si se 
toma para la luz una longitud de onda de $0,550 \mu \mathrm{m}$ y la distancia al punto próximo para el ojo es de 25 $\mathrm{cm}$, las ecuaciones (1), (2) y (3) conducen a los siguientes resultados:

$$
\begin{aligned}
& f=\frac{1,5 \times 0,8 \mathrm{~mm}}{4 \times(1,5-1)}=0,6 \mathrm{~mm}, \\
& M=\frac{25 \mathrm{~cm}}{0,06 \mathrm{~cm}} \simeq 417, \\
& r_{\mathrm{A}}=\frac{1,22 \times 0,550 \mu \mathrm{m} \times 0,6 \mathrm{~mm}}{0,6 \mathrm{~mm}} \simeq 0,7 \mu \mathrm{m} .
\end{aligned}
$$

\section{Resultados y conclusiones}

La elección de una esfera de vidrio de tan pequeño diámetro hace posible que el microscopio supere los 400 aumentos; más que los microscopios simples tomados como referencia histórica (de 20 a 160 aumentos en los microscopios de Bancks o 266 aumentos en el más potente microscopio de Leeuwenhoek conservado).

Como se ha señalado anteriormente, la mala calidad atribuida a los microscopios simples por culpa de las aberraciones ópticas es una creencia que se desmonta fácilmente con las observaciones, incluso con un instrumento de alta potencia como este que nos ocupa.

Para poner a prueba la bondad del microscopio se ha elegido una preparación de sangre humana, teñida con hematoxilina-eosina. Después, se ha fotografiado la misma zona con el microscopio simple y con un microscopio compuesto; en este caso a través de un objetivo Leitz acromático de 40 aumentos y ocular de 15 aumentos (figura 8). Con el microscopio simple se reconocen claramente los eritrocitos (su diámetro se encuentra en torno a $7 \mu \mathrm{m}$ ) y un leucocito con núcleo multilobulado, aunque la imagen empeora hacia la periferia del campo de visión.

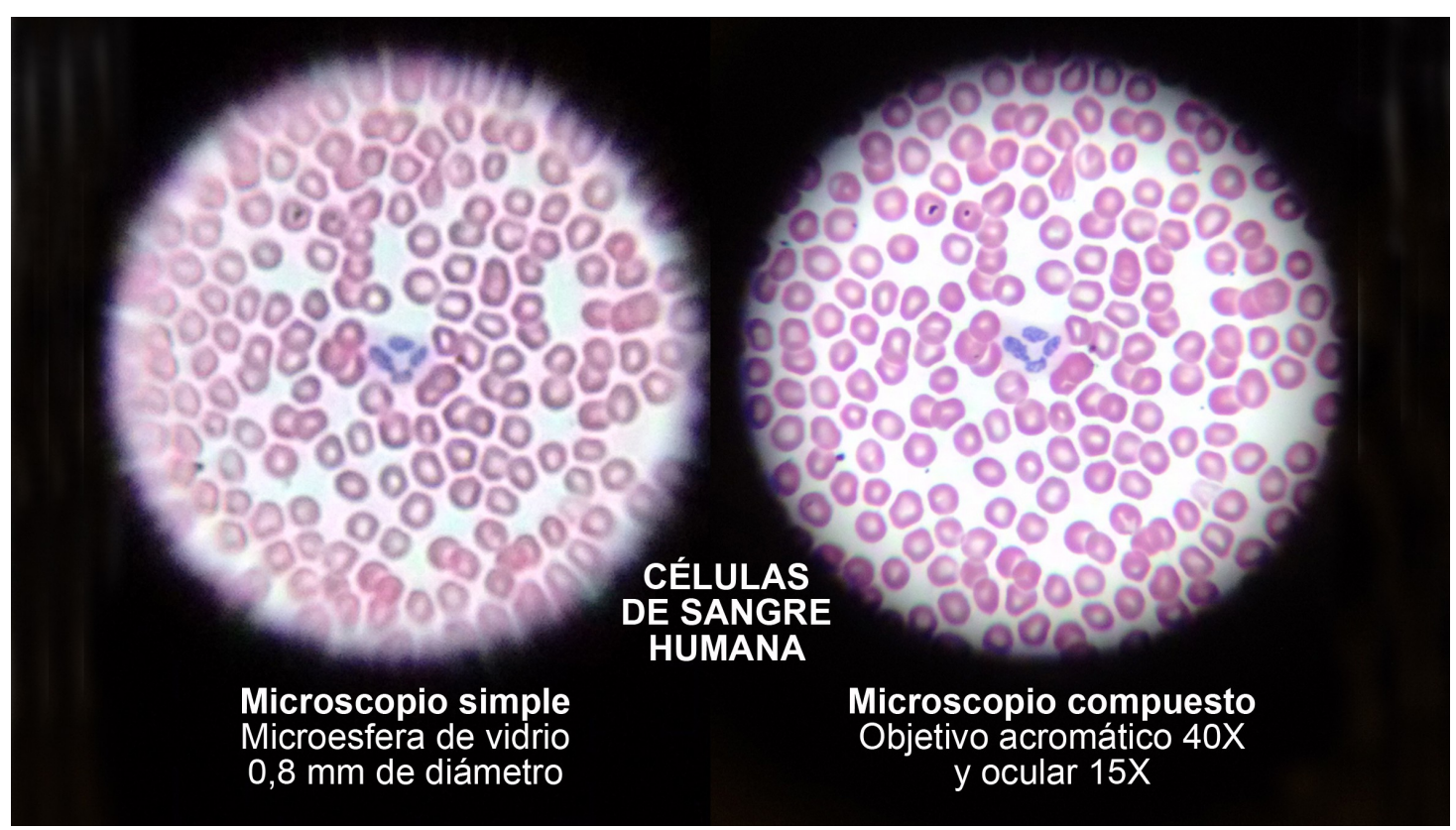

Figura 8. La misma zona de una preparación microscópica de sangre humana, fotografiada con la microesfera del microscopio simple y con el objetivo acromático de un microscopio compuesto. 
Del valor obtenido para el radio de Airy se deduce que, teóricamente, este microscopio simple podría resolver detalles de hasta $0,7 \mu \mathrm{m}$. El cálculo ignora el efecto de aberraciones ópticas tales como la cromática y la esférica.

Una estimación razonable de la resolución puede hacerse examinando bacterias. La descomposición de hojas de lechuga mantenidas en agua durante varios días conduce a una gran proliferación bacteriana. Del vídeo grabado a través del microscopio (Del Mazo 2018), que muestra en vivo la población de bacilos de una gota de agua, se ha seleccionado una imagen (figura 9). Sabiendo que los bacilos tienen diámetros próximos a $1 \mu \mathrm{m}$, puede concluirse que el microscopio resuelve objetos de este tamaño, algo superior al calculado teóricamente.

Con todo esto, se pone de manifiesto que el microscopio simple no es solo un instrumento curioso carente de utilidad real; su calidad puede igualar o superar a la de los microscopios compuestos más básicos.

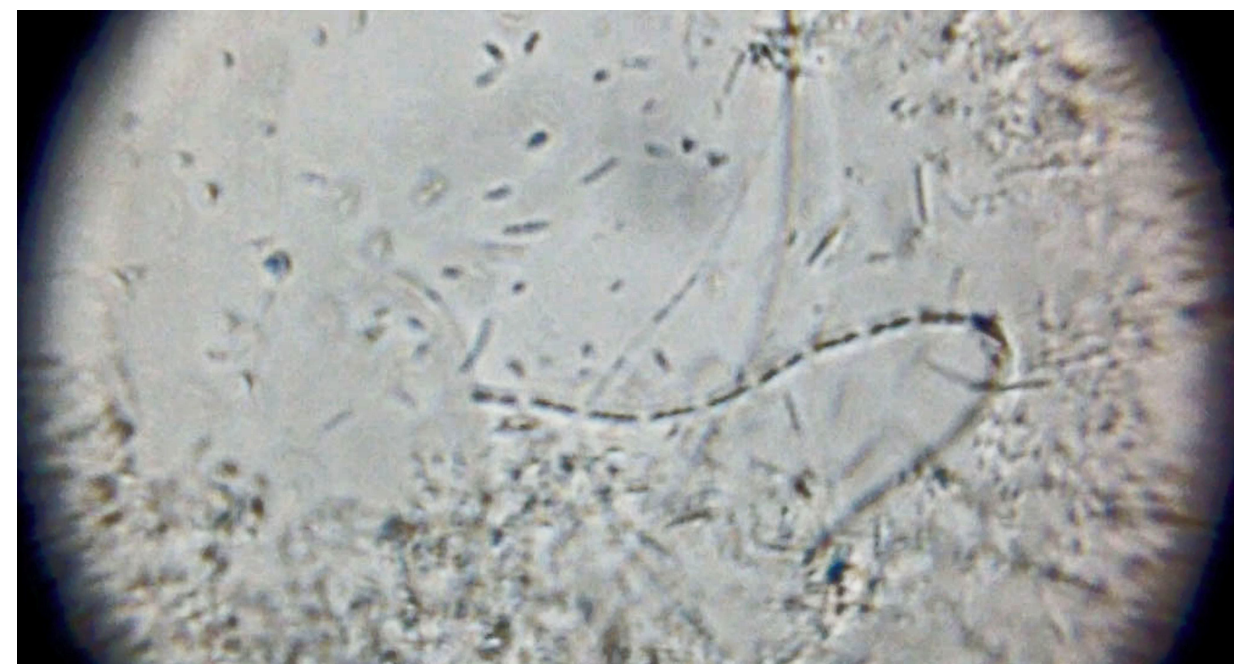

Figura 9. Bacilos desarrollados tras mantener en agua hojas de lechuga durante varios días. Estos microorganismos tienen un diámetro próximo a $1 \mu \mathrm{m}$. La imagen pertenece a un vídeo grabado a través del microscopio del autor (Del Mazo 2018).

En 2014, Manu Prakash, bioingeniero de la Stanford School of Medicine, desarrolló el foldscope (Cybulski 2014). Se trata de un microscopio simple muy barato pensado para que, aquellos lugares del mundo con escasos recursos económicos, puedan contar con un instrumento eficaz en el diagnóstico de enfermedades como la malaria. Aunque con este microscopio se pueden conseguir hasta 2000 aumentos cuando trabaja con microesferas de zafiro de $0,2 \mathrm{~mm}$ de diámetro, la versión que se comercializa lleva una bola de borosilicato de 2,4 $\mathrm{mm}$ de diámetro, que proporciona 140 aumentos, con una resolución de $2 \mu \mathrm{m}$. Esto corresponde a un microscopio simple de potencia media, válido para un rango de observaciones diferente al del microscopio de alta potencia que aquí se ha tratado.

Con las microsferas usadas en señalización vial, cuyos diámetros no superan el milímetro de diámetro, solo pueden construirse microscopios de grandes aumentos, adecuados para observar, por ejemplo, pequeños organismos unicelulares u orgánulos celulares. Si se quieren construir microscopios que trabajen con menos aumentos pueden utilizarse esferas de vidrio de buena calidad óptica con diámetros comprendidos, por ejemplo entre $1,0 \mathrm{~mm}$ y $3,0 \mathrm{~mm}$, que pueden adquirirse a través de internet. Una solución completamente diferente para fabricar microscopios que no superan los 50 aumentos, consiste en sustituir las esferas de vidrio por las lentes colimadoras biconvexas con que cuentan los pequeños punteros láser, tan extendidos actualmente. 
Es necesario decir que, tanto el montaje de la microesfera en un soporte rígido como el del sistema de enfoque, requieren un trabajo cuidadoso; no obstante, a pesar de que el dispositivo funciona satisfactoriamente, es posible que puedan encontrarse otras soluciones mecánicas más sencillas.

Procedencia de las ilustraciones. Todos los dibujos, figuras explicativas y fotografías que aparecen en el artículo han sido realizadas por el propio autor, quien ha preparado también las muestras microscópicas que han servido para la toma de las micrografías.

\section{Referencias bibliográficas}

Brown R. (1828) A brief Account of Microscopical Observations made in the Months of June, July, and August, 1827, on the Particles contained in the Pollen of Plants; and on the general Existence of active Molecules in Organic and Inorganic Bodies. The Philosophical Magazine and Annals of Philosophy 4(21), 161-173

Cybulski J. S., Clements J., Prakash M. (2014) Foldscope: Origami-Based Paper Microscope. PLoS ONE 9(6), e98781.

Del Mazo A. (2018) Microscopio simple (vídeo en YouTube).

Dobell C. (1932) Antony van Leeunenhoek and his "Little animals". New York: Harcourt, Brace and Company.

Ford B. (1985) Single Lens: The Story of the Simple Microscope. New York: Harper \& Row.

Hecht E. (2000) Óptica. Madrid: Addison Wesley Iberoamericana.

Hooke R. (1989) Micrografía. Madrid: Alfaguara.

Robertson L. A. (2015) Van Leeuwenhoek microscopes - where are they now? FEMS Microbiology Letters 362(9), fnv056.

Snyder L. (2017) El ojo del observador. Johannes Vermeer, Antoni van Leeuwenhoek y la reinvención de la mirada. Barcelona: Acantilado

Stafford B., Terpak F., Poggi I. (2001) Devices of Wonder: From the World in a Box to Images on a Screen. Los Ángeles: Getty Research Institute.

Zuylen J. (1981) The microscopes of Antoni van Leeuwenhoek. Journal of Microscopy 121(3), 309-328. 